\title{
Deploying a Model for Assessing Cognitive Automation Use Cases: Insights from Action Research with a Leading European Manufacturing Company
}

\author{
Christian Engel \\ University of St. Gallen, Switzerland \\ christian.engel@unisg.ch
}

\author{
Edona Elshan \\ University of St. Gallen, Switzerland \\ edona.elshan@unisg.ch
}

\author{
Philipp Ebel \\ University of St. Gallen, Switzerland \\ philipp.ebel@unisg.ch
}

\begin{abstract}
Cognitive automation moves beyond rule-based automation and thus imposes novel challenges on organizations when assessing the automation potential of use cases. Thus, we present an empirically grounded and conceptually operationalized model for assessing cognitive automation use cases, which consists of four assessment dimensions: data, cognition, relationship, and transparency requirements. We apply the model in a real-world organizational context in the course of an action research project at the customer service department of ManuFact $A G$, and present unique empirical insights as well as the impact the application of the model had on the organization. The model shall help practitioners to make more informed decisions on selecting use cases for cognitive automation and to plan respective endeavors. For research, the identified factors affecting the suitability of a use case for cognitive automation shall deepen our understanding of cognitive automation in particular, and AI as the driving force behind cognitive automation in general.
\end{abstract}

\section{Cognitive Automation: Moving Beyond Rule-Based Automation}

Due to steep technological innovations in algorithms, computing power, and data storage that led to the (renewed) rise of Artificial Intelligence (AI) during the last decade, we are now witnessing an increasing interest in a phenomenon known as "cognitive automation". This phenomenon refers to automating or augmenting tasks and processes seizing inference-based algorithms in order to process structured and unstructured data leading to probabilistic outcomes [20]. In that, cognitive automation moves beyond "classic", rule-based automation, enabled by generic IT, as it takes over certain degrees of cognition by providing two types of outputs - decisions and/or solutions [19]. To "produce" decisions or solutions, cognitive automation uses AI, mostly in the manner of Machine Learning, which operates in an inference- based and probabilistic manner $[12,20]$. Thus, it is viewed to be the next evolutionary step beyond rulebased, deterministic automation approaches such as the prominent application of so-called robotic process automation (RPA) [20,33].

For companies, cognitive automation offers opportunities for gaining competitive advantage in various ways [35]: First, from a process-perspective, companies can further increase their levels of automation or reengineer their processes such as deploying voice bots for handling customer requests.

Second, organizations can seize cognitive automation to develop completely new or enhanced products and services such as autonomous vehicles that perceive the environment, reason about it and accordingly take over certain degrees of decisionmaking regarding respective driving maneuvers.

Third, organizations can use cognitive automation to derive insights from various data sources, and even Big Data to aid and/or automate decision making, and to transform an organization's strategy such as seizing cognitive automation for timely sensing and reacting to external risks and threats (e.g., global pandemics).

Thus, cognitive automation is a strategic enabler of business transformation and productivity improvements beyond mere cost-cutting initiatives [20]. This potential has already been recognized in practice. 75 percent of technology and operations executives stated in a survey among 550 participants from 2019 to expect it to have "meaningful impact on their business performance within the next three years" [12, p.25] and 70 percent agree that it will lead to higher-value work for employees [12].

\section{The Need for a Cognitive Automation Use Case Assessment Model}

As cognitive automation is based on inference, which leads to probabilistic outcomes, respective endeavors are rather experimental and perceived to be risky by organizations. This is also reflected in 
organizational adoption rates. So far only 26 percent of potential adopter organizations state to have particular systems in place, which is rooted in the still comparably high price of cognitive automation tools, the required amounts of data, and the insecurity of organizations due to the unpredictability of outcomes [21]. Further exemplary reasons for this are covered by what Lacity and Willcocks [20, p.95] call the "wrenches of cognitive automation": the data wrench, the algorithm wrench, and the technology embeddedness wrench [20]. In that, organizations are struggling to acquire and handle the vast amounts of data that are needed to train cognitive automation tools and face issues such as dark, difficult, incorrect, duplicate, inconsistent, or outdated data [11, $20,28]$. These data-related issues are viewed to be one of the key challenges for future automation technologies [11]. From an algorithmic perspective, two exemplary challenges for organizations are the transferability of learning between domains, and the explainability issue of algorithms that leads to the black box character of AI. Finally, from an IS perspective, the technology embeddedness wrench refers to the required organizational changes that need to be induced into socio-technical systems [20].

The relevance for practice-based insights in this research vein is also reflected when analyzing related research in this field: The question of what should be automated and what should be done by humans is not new [1]. If organizations select a task or process that is not suited for automation, the endeavor is likely to fail, which demands structured approaches for assessing and selecting tasks or processes to increase the likelihood of success [5, 22]. Thus, researchers have investigated the selection of suitable automation use cases. For instance, research has identified use case characteristics, such as task complexity [13], distinguishing routine versus nonroutine and manual versus cognitive tasks [4], or deduced the automation potential of use cases from their required skills such as perception and manipulation, creative intelligence, or social intelligence [17].

Recent research on selecting automation candidates has been driven by the emergence of RPA during the last years [33]. For instance, new models have been developed to select suitable automation candidates for RPA (e.g., [22]). These models build upon assessment criteria developed for these purposes. RPA is recommended when levels of standardization, maturity, transaction volume, and existence of business rules are high [20]. According to other criteria, rule-based routine tasks with few exceptions, and little or no cognitive reasoning are most suitable for RPA [3] - to name a few. Thus, we can summarize that selecting the right automation use cases - be it single tasks or whole processes - constitutes an essential step in deciding on automation endeavors and has attracted attention from both researchers and practitioners.

However, as cognitive automation moves beyond rule-based automation, we face a different degree of richness in the scientific knowledge base. Due to the rather experimental character, which is rooted in the probabilistic outcomes "produced" by cognitive automation solutions, the need for such systems to learn from data, and the particular socio-technical challenges such as fear of job loss, we argue that the models and sets of criteria developed for rule-based automation (see above) do not cover cognitive automation as it is a phenomenon of perceiving, reasoning, and inferring. This is reflected in the following quote from automation research: "More procedural or predictable tasks are handled by smart machines, while humans have become responsible for tasks that require inference, diagnoses, judgement, and decision making" [26, p.1619].

A lot of these assumptions do not hold nowadays due to the technological developments in the realm of AI. However, to the best of our knowledge, there does not exist a model for assessing cognitive automation use cases that is tailored to this novel phenomenon.

\section{A Model for the Assessment of Cognitive Automation Use Cases}

We first explain how we developed our model for assessing cognitive automation use cases before we present the model and its particular components.

\subsection{Model Development}

In order to develop a model for assessing cognitive automation use cases, we first have to identify the relevant dimensions along which a cognitive automation use case can be characterized in terms of the requirements its characteristics impose on a cognitive automation endeavor. This set of assessment dimensions shall allow for generalizability and hold across various organizational contexts.

As theoretical research in this vein is still nascent, we induce the dimensions from the organizational context. Thus, we seized the technique of semistructured interviews [24] with practitioners from the field. We purposefully selected the interviewees to achieve a high level of variation. To still maintain comparability between the interviews, the interviewees were selected from representatives of large corporations, who were involved in cognitive automation projects.

Over the duration of one year (03/2019 - 03/2020), we interviewed 17 company representatives from various industries, who were involved in nine cognitive 
automation projects and were based on different hierarchy levels of the organizations (Table 1). This allowed us to establish a comprehensive understanding of the dimensions affecting cognitive automation endeavors. The semi-structured interviews followed a predefined guideline but allowed for naturally flowing conversations through incorporating variations in topics and adaptability to emerging themes [24]. During the interviews, we asked the interviewees about assessment criteria for selecting cognitive automation use cases in general, and about the tasks and processes that were subject to cognitive automation, their reasoning for selecting the latter, and the efforts and risks that were faced in the projects.

Based on the interview transcripts, two researchers extracted data from the material and engaged in open, axial, and selective coding [16]. After openly coding the documents and assigning relationships among the open codes (axial coding), we set the core variable for selective coding to be "requirements dimensions of use case characteristics" to identify factors that need to be assessed to determine the degree to which a use case is suitable to cognitive automation. We iteratively evaluated the coding in discussions among two researchers to reach validity and reproducibility [16].

Table 1. Interview Information

\begin{tabular}{|c|c|}
\hline $\begin{array}{l}\text { Project / } \\
\text { Industry }\end{array}$ & $\begin{array}{c}\text { Positions of Interviewees } \\
\text { (Number of Interviews / Total Duration) }\end{array}$ \\
\hline \multirow{3}{*}{$\begin{array}{c}\text { Alpha / } \\
\text { Telecom- } \\
\text { munication }\end{array}$} & Capability Management Head (1/30 min.) \\
\hline & Project Owner from Business (1/60 min.) \\
\hline & Project Manager (1/60 min.) \\
\hline \multirow{2}{*}{ Beta / Banking } & Chief Information Officer (1/40 min.) \\
\hline & Project Manager (2/120 min.) \\
\hline \multirow{2}{*}{\begin{tabular}{c|} 
Gamma / \\
Manufacturing
\end{tabular}} & Chief Information Officer (1/30 min.) \\
\hline & Project Manager (2/80 min.) \\
\hline \multirow{3}{*}{$\begin{array}{c}\text { Delta / } \\
\text { Banking }\end{array}$} & Head of Data and Analytics (1/40 min.) \\
\hline & Head of Platform Strategy (1/35 min.) \\
\hline & Project Manager (2/120 min.) \\
\hline \multirow{2}{*}{$\begin{array}{c}\text { Epsilon / } \\
\text { Manufacturing }\end{array}$} & Vice President IT Innovation (1/50 min.) \\
\hline & Project Manager $(1 / 50 \mathrm{~min})$. \\
\hline \begin{tabular}{c|} 
Zeta / \\
Automotive \\
\end{tabular} & $\begin{array}{c}\text { Executive Manager AI Strategy and } \\
\text { Architecture (1/110 min.) }\end{array}$ \\
\hline \multirow{2}{*}{$\begin{array}{c}\text { Eta / } \\
\text { Manufacturing }\end{array}$} & Project Manager Business (1/45 min.) \\
\hline & Project Manager Legal (1/45 min.) \\
\hline Theta / Pharma & Senior Data Scientist (1/120 min.) \\
\hline Iota / Insurance & Chief Data Officer (1/120 min.) \\
\hline
\end{tabular}

The resulting model consists of a structured set of questions that operationalize respective sub-constructs and items to grasp the four assessment dimensions. These were deduced from literature to enrich the induced practice-oriented dimensions with conceptual clarity from research.

To conduct a first evaluation cycle of the model in terms of exhaustiveness, understandability, and potential utility for practice, we drew on a focus group [24] consisting of six participants from the interview study, which led to a positive evaluation of the model.

\subsection{Components of the Assessment Model}

Figure 1 provides an overview of the developed assessment model and the constructs used to operationalize it for further testing and refinement in practice. We briefly summarize the assessment dimensions of use case characteristics that resulted from the iterative coding of the interview transcripts. These are purposefully positioned as requirements dimensions to facilitate the translation between use case characteristics and the implications for cognitive automation endeavors, which shall serve as a mediator between business and IT departments. If these requirements are high (low), this means that the level of effort or difficulty of implementing a use case with cognitive automation will be high (low). Thus, the cognitive automation suitability of a use case is rather a continuous degree than a dichotomy.

\begin{tabular}{|c|c|c|c|}
\hline & \multicolumn{2}{|c|}{$\begin{array}{l}\text { Suitability of Cognitive } \\
\text { Automation Use Case }\end{array}$} & \\
\hline \multicolumn{4}{|c|}{ Cognitive Automation Use Case Assessment Dimensions } \\
\hline $\begin{array}{c}\text { Data } \\
\text { Requirements } \\
\end{array}$ & $\begin{array}{c}\text { Cognition } \\
\text { Requirements } \\
\end{array}$ & $\begin{array}{l}\text { Relationship } \\
\text { Requirements }\end{array}$ & $\begin{array}{l}\text { Transparency } \\
\text { Requirements }\end{array}$ \\
\hline$--\frac{1}{(+)}$ & $--\frac{1}{(+)}$ & $f--$ & $--f \frac{1}{(+)}$ \\
\hline $\begin{array}{l}\text { Data Integrity } \\
10,27] \\
\text { Value-Added [32] } \\
\text { Accessibility [32] } \\
\text { Amount of Data } \\
32] \\
\text { Accuracy } \\
10,14,32] \\
\text { Completeness } \\
10,14,32] \\
\text { Consistency } \\
10,14,32] \\
\text { Datedness } \\
10,14,32] \\
\text { Interpretability } \\
10,14,32]\end{array}$ & $\begin{array}{l}\text { Complexity as a } \\
\text { Proxy [13,23]: } \\
\text { - Size } \\
\text { - Variety } \\
\text { - Ambiguity } \\
\text { - Relationships } \\
\text { - Variability } \\
\text { - Reliability } \\
\text { - Novelty } \\
\text { - Incongruity } \\
\text { - Action } \\
\text { Complexity } \\
\text { - Temporal } \\
\text { Demand }\end{array}$ & $\begin{array}{l}\text { - User Partici- } \\
\text { pation Mode [6] } \\
\text { - Organizational } \\
\text { Response [15] } \\
\text { - Timeliness } \\
\text { - Facilitation } \\
\text { - Redress } \\
\text { - Apology } \\
\text { - Credibility } \\
\text { - Attentiveness }\end{array}$ & $\begin{array}{l}\text { - Audit Require- } \\
\text { ments [8] } \\
\text { - Transparency } \\
\text { Stakeholders [18] } \\
\text { - Transparency } \\
\text { Meaningfulness } \\
\text { [18] } \\
\text { - Transparency } \\
\text { Usefulness [18] } \\
\text { - Information } \\
\text { Quality in Trans- } \\
\text { parency [18] }\end{array}$ \\
\hline
\end{tabular}

Figure 1. Cognitive Automation Use Case Assessment Model

Data requirements of a use case refer to the need for a cognitive automation solution to acquire, store and access data about the task or process input entities, the respective task or process outputs that shall be created, as well as the use case context. In that, required use case data needs to be gathered and processed to information, ultimately resulting in knowledge about how a task or process should be carried out [2]. This induces challenges that are use case-specific and vary with the degree of data quality, which is widely defined as fitness for use [14]. Our interview data illustrates this point. 
"Data quality and what it takes to source the data has been underestimated. If the sourcing of data is intensive, then there is always a lot of pre-investment before something comes back and it is more difficult to convince the organization." - Head of Capability Management (Alpha)

To operationalize the dimension of data requirements, we draw on established constructs from literature. In that, a use case varies along the requirements imposed by the level of data integrity, accuracy, completeness etc. (see Figure 1) [10, 14, 27, 32].

Cognition requirements of a use case refer to the needs that a task or process imposes on the capabilities of a cognitive automation tool with regards to entity perception, learning, reasoning, and interacting. These capabilities are related to knowledge acquisition and understanding through thought. Transferring decision making or creation of resolutions from humans to machines implies the necessity of a machine to reconstruct the cognitive capabilities required to conduct a task [30], which is also reflected in the following exemplary quote from the interviews.

"[T] here needs to be a decision capability. [...] The heuristics in our brain are probably not completely decoded in algorithms so far. So, that is where the challenge would be." - Project Manager (Delta)

Thus, this assessment dimension is linked to the construct of task complexity as a complex task has been defined as one, which imposes high cognitive requirements on a task agent [13]. In that, task complexity is operationalized through the constructs of size, variety, ambiguity of task etc. (see Figure 1) [13, $23]$.

Relationship requirements of a use case pertain to the degree to which a cognitive automation tool needs to perceive and/or form social or professional bonds during task or process performance. This assessment dimension is reflected in the theory of social presence [31]. In that, machines face several challenges in conveying social cues in the same manner as humans do [25], which is linked to research that focuses on how anthropomorphic features and behavior of machines affect the relation of a human towards machine agents [29]. The following quote summarizes this dimension.

"[W] cannot completely interfere or break the way of interaction with our users. So, we are looking for solutions that can seamlessly be integrated with the current logic and what people are used to. [We] just take over the cases that are good fit for automation." Vice President IT Innovation (Epsilon)

The requirements are operationalized through the required level of user participation [6], and organizational response (see Figure 1) [15].
Transparency requirements of a use case are defined as the degree to which a cognitive automation tool needs to be capable of understanding and explaining what happens between task/process inputs and outputs. This assessment dimension relates to the research vein of "explainable AI", which investigates the tradeoff between the accuracy of cognitive machines and their explainability [9]. Thus, developers of cognitive systems face the challenge to design their systems in a way of being performant while allowing for the necessary transparency level [34].

"The auditability in the sense of explainable AI must be checked, e.g. when driving autonomously. I can't let the car drive if I cannot say why it drove over this pedestrian." - Executive Manager AI Strategy and Architecture (Zeta)

For operationalization, we use constructs from literature such as audit requirements [8], and the distinct types of transparency such as in relation to stakeholders, meaningfulness etc. (see Figure 1) [18].

\section{Assessing the Use Case of Cognitive Automation in Customer Support at ManuFact AG}

In this section, we show how we applied the developed model in the course of an action research project together with a large manufacturing firm - called ManuFact AG for the purpose of anonymity at this stage of our research.

ManuFact AG was not in the sample of companies that served as an empirical basis for constructing the assessment model presented in the previous section. This setup is chosen on purpose to facilitate an objective application of the model without confirmation bias. We rather pursue the goal to showcase the applicability of the model for assessing cognitive automation use cases in a real-world context, where its application has actual consequences on managerial decisions that are made on the future pathway of the desired cognitive automation endeavor within ManuFact AG.

\subsection{Case Description and Drivers of Cognitive Automation at ManuFact AG}

ManuFact $A G$ is one of the market leading manufacturing companies in the realm of sanitary products in Europe, and operates on a global basis. To distribute its high-quality sanitary products, the company uses a three-stage distribution model. This includes direct distribution to wholesalers, planners, and plumbing firms. Private customers then purchase the products indirectly via these sales channels. As ManuFact AG offers a wide range of distinct sanitary 
products, which are available in a variety of combinations of distinct components and designs, the complexity of the product portfolio is high. To achieve a high level of service quality for both their B2B customers (i.e., the indirect sales channels) and the actual users of the products (i.e., B2C customers), ManuFact AG provides two helplines for handling both questions from the $\mathrm{B} 2 \mathrm{~B}$ professionals and also a helpline for $\mathrm{B} 2 \mathrm{C}$ customers. We focus on the $\mathrm{B} 2 \mathrm{~B}$ helpline in this paper but relate it to the $\mathrm{B} 2 \mathrm{C}$ side whenever this is necessary as there are requests that are forwarded between the lines.

In the B2B helpline, which is intended to serve the needs of professionals from the sanitary industry, subject matter experts (called helpline experts in this paper) with year-long experience in the field and an educational background as Master Sanitary Engineers work to achieve the highest level of customer support and service quality possible. In this regard the company is leading in the level of service it offers in the industry. This is reflected in the following quote.

"After all, we have cisterns that are 50 years old. Our cisterns just won't break. We guarantee 25 years of spare parts safety and we can also fulfil customer requests for these older products. In this respect we are really ahead of the market, nobody else in the market can do this. "-Helpline Head

Through three channels - email, phone, and live chat - the helpline experts handle over 400,000 customer requests per year. These range from requests regarding the provision of user manuals, marketing material, or product identification numbers to highly complex questions regarding sanitary installations, and even topics such as fire protection.

As the service level has been on a consistently high level in the past, the expectations of the customers are high as these are used to a highly professional, fast and friendly consultation and problem resolution provided by ManuFact AG.

"Speed and competence are very important. There are people who call my number directly again and again. We know them by name by now. And we always have good feedback." - Helpline Expert 1

However, during the last years several developments increasingly pose challenges with regards to maintaining this high level of service quality without increasing in department size.

First, the number of requests that reach the helpline experts through written channels such as emails has increased by 23 percent in 2019 compared to 2018 . Overall, a shift from calling via phone to more written communication by the customers can be observed as a trend.

Second, since the deployment of the live chat in 2019, the multi-channel communication that sometimes happens simultaneously through various channels (e.g. live chat and phone at once with different customers) poses a challenge.

Third, the increasing complexity of the product portfolio drives and increases the number of requests and the time needed for resolving them. In total, the number of requests has increased by 10 percent in 2019 compared to 2018.

Fourth, due to the rise of online wholesalers and doit-yourself trends, for instance driven by social media, the number of requests that reach the wrong helpline (i.e., B2C customers that end up in the professional B2B helpline) has increased.

To maintain the same high level of service quality and their role as a service leader in the sanitary industry in the future, ManuFact AG thus intends to assess the use case of augmenting their helpline with cognitive automation tools.

\subsection{Action Research Mode}

Despite from seizing the potential of cognitive automation for augmenting their helpline employees' work, ManuFact AG intends to position a potential future project as a lighthouse project with organizational-wide impact to showcase the use of cognitive automation tools as a trigger of respective initiatives. Thus, ManuFact AG regards an initial use case assessment as a necessary step to find out if the use case is suitable for cognitive automation, and to increase the likelihood of project success.

Therefore, an action research team [7] is set up consisting of two researchers that developed the assessment model described above and two project managers from the company - one from the IT and one from the business department where the helpline is hosted. Both have year-long experience in project management and basic to advanced skills in analytics and Machine Learning.

We present the action research mode along the five phases of the action research cycle [7]: (1) diagnosing, (2) action planning, (3) action taking, (4) evaluation, (5) specifying learnings.

Accordingly, the core team of two researchers and the two project managers from ManuFact AG engaged in (1) diagnosing and (2) action planning [7], i.e. planning the mode of how the assessment of the cognitive automation use case should be carried out. As the model consists of a closed set of standardized questions, it was agreed to extend the set of questions with an introductory section to better contextualize the assessment and to take into account the organizational specificities, the needs, and the professional background of the interviewees. 
During the (3) action taking phase [7], the two researchers interviewed four helpline experts, including the helpline head, who is also involved in operational work, in structured two hour-interview sessions. The interviews were conducted separately with each helpline expert to prevent bias potentially rooted in psychological peer pressure or more dominant interview participants. The responses were documented in the developed framework by two researchers, and aggregated by removing duplicates. Additionally, document analysis of exemplary customer requests, job profiles of the helpline experts, and performance reports of the helpline over the last years was conducted. Furthermore, the researchers contacted the helpline themselves with a scripted professional request that was provided by ManuFact AG in order to experience the process themselves, and to contextualize the interview insights. This was combined with an on-site visit of the helpline offices, which allowed the researchers to observe the work mode of the helpline experts in their environment.

In the (4) evaluation phase [7], after iterative discussions among the core project team, a review workshop was conducted with the interviewed helpline experts and the core project team to clarify whether the documented interview insights were correct and if anything was missing.

Afterwards, the gathered data was completed with the additional insights, and the assessment results were retrieved by analyzing the data structured along the four assessment dimensions of the model to (5) specify the learnings [7]. In this course, the researchers iteratively paired the insights from the interviews, document analysis, and on-site observations with the respective constructs and items of the requirements dimensions. Furthermore, they indicated whether a particular construct or item leads to an increase in use case requirements of the planned cognitive automation endeavor or not.

\section{Results of the Use Case Assessment}

An overview of the assessment of the use case of implementing cognitive automation in the $\mathrm{B} 2 \mathrm{~B}$ helpline of ManuFact AG is provided in Figure 2.

The overall assessment of the use case shows that it varies widely between the distinct assessment dimensions. In a nutshell, the use case can be described as being data-intense, transparency-loose, but volatile in the dimensions of relationship and cognition requirements depending on its concrete sub-use case specifications. We present the insights (I1-I18) that are most significant for shaping our overall assessment along the dimensions of the model.

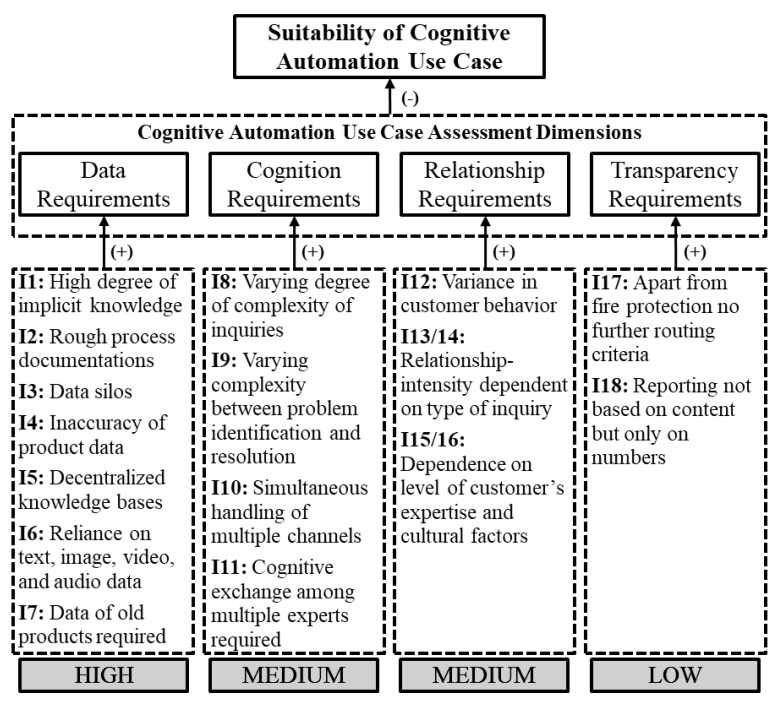

\section{Figure 2. Overall Results of the Use Case} Assessment at ManuFact AG

Data requirements: Overall, the data requirements were found to be high in this use case as there is a high level of undocumented knowledge and processes, and a high degree of distributed and implicit experience knowledge. Furthermore, the helpline experts have to process images, videos, and even audio data as essential data sources to handle a large share of the requests. This means that in any case, this requirement dimension induces high efforts into a potential project. Thus, prework is necessary prior to an actual cognitive automation project. The following insights lead to this assessment outcome in the dimension of data requirements.

In the customer helpline of ManuFact AG, work is based on the experience-based knowledge of the helpline experts. Additionally, a large part of the knowledge is not explicitly written down but implicitly present in the heads of the employees (I1). This is reflected in the following quote by the Helpline Head of ManuFact AG.

"This basic knowledge is demanded and required. The employee answers the simple questions directly from the hip, because he or she has the information stored on their 'disk'." - Helpline Head

Thus, the large amount of implicit experience knowledge would have to be systematically recorded beforehand to make the data machine-readable.

Furthermore, process documentations, which determine how customer enquiries are dealt with, only exist in rough resolution (I2). Helpline Expert 3 summarizes this as follows:

"How we work and how we formulate our replies, is not documented and we do it out of habit." - Helpline Expert 3 
For preparing a cognitive automation initiative, processes of frequently occurring requests should be jointly documented and standard answers or text modules for frequent requests should be developed.

In this vein, even though knowledge transfer and data exchange take place within the helpline department, there exist data silos in relation to other departments (I3). This is made graspable with the following quote.

"The Excel is read-only, so it's not for anyone to work on it. There are numbers in the Excel files that you can't find anywhere else." - Helpline Head

In that, the helplines' databases are protected, but this leads to a high volume of requests from other departments. A mutual exchange with other departments (e.g., product managers) should be initiated in order to store and maintain the required knowledge in an institutionalized manner accessible to all relevant stakeholders.

These silo effects also concern product data, which is not up-to-date and thus poses a challenge for the helpline. One reason for this is that there exists a time delay in communication between branches in different countries (I4). For example, changes to documentation or product specifications by the product managers sometimes do not appear at all or are communicated to the helpline significantly delayed, as indicated by Helpline Expert 2.

"Some of the measurements in the technical drawings are wrong. These will be eventually corrected at some point in time." - Helpline Expert 2

This requires an interdepartmental agreement before launching a cognitive automation initiative on how data is shared and kept up-to-date in the organization.

Also, within the helpline department knowledge is stored in individual databases in a distributed manner (I5). This means that in addition to a central data drive within the department, employees store their knowledge decentralized in individualized Excel sheets. The individual reasons for this are described by Helpline Expert 2.

"You will then find your way better on your own drive and don't have to search so long on the centralized department drive. You can't know everything; you just have to know where it is. " - Helpline Expert 2

Thus, a common collection of the best components of individual knowledge documentation should be established to prepare a cognitive automation project.

Regarding the types of data, the helpline work necessarily relies on unstructured and heterogeneous data (I6). The required information is not only available in written form, but also as images, videos and audio files. The importance of these data sources is emphasized by the Helpline Head.
"Pictures are worth a thousand words. [...] We actively ask customers to send us pictures, and there are video clips too. They are standard, even with sound, such as flow sounds." - Helpline Head

Cognitive automation serves as a means to process structured and unstructured data and thus can be highly effective in this case. However, a training data set consisting of text, images, videos, and audio files will have to be set up and interlinked between the distinct data types, which will be effortful considering the broad spectrum of products.

Furthermore, due to the high quality of the products, their age and the long service life affect daily work (I7). As the customers and the helpline need product information longer than it is available on the website, this causes challenges in identifying older products a customer might be referring to.

"There are also products that were produced 15 years ago." - Helpline Expert 1

For the preparation of a cognitive automation project, a database should be developed, which automatically archives the product information of the current website over time.

Cognition requirements: Overall, the cognition requirements were found to be on an intermediate level, however, exhibiting a high level of volatility as the complexity of customer requests varies greatly depending on customer requirements and types of requests. As a consequence, sometimes problem identification and sometimes problem solution are more challenging for the helpline experts. Thus, a cognitive automation solution needs to be able to recognize and classify these cases. The following insights substantiate this assessment.

Customer enquiries are very individual due to the "human factor", and differ significantly in their degree of complexity (I8). Although, there are inquiries, which occur more frequently, the one standard customer inquiry does not exist. For instance, as indicated by the following quote, this leads to significant variations in the time required for handling a customer request.

"The more complex the inquiry, I may be off the line for half an hour and then do research." - Helpline Expert 1

An automatic subdivision into simple and complex requests through cognitive automation can increase the plannability of the task. However, the cognitive system needs to be trained accordingly to exhibit the required cognitive capabilities for this.

In addition, the identification of the product and the initial understanding of the problem can be particularly challenging (I9), as underlined by Helpline Expert 1.

"The hardest part is when you have a request and need to identify what product it is and what the problem is. " - Helpline Expert 1 
Especially older products, inaccurate information from customers and the wide range of products increase the complexity of the task. In most cases, the problem identification task is the one that takes the most time. The problem solution can then usually be worked out relatively quickly. Separating the problem identification- from solution creation-intense sub-use cases will induce effort into a prospective cognitive automation project.

As indicated earlier, a challenge within the helpline department is the situation that sometimes two different channels have to be processed simultaneously, which is a cognitive stress factor for employees (I10). I.e., the live chat and the telephone are sometimes processed simultaneously. The consequences of this are described by Helpline Expert 2.

"The timing of calls and chat is determined by the client. I can clock the emails myself. It can happen that I'm on the phone and there comes a chat and then I have to do both." - Helpline Expert 2

An automation of the initial reception of chat requests and structured querying of the request and customer data is conceivable and thus can serve as a further sub-use case within a future project.

Finally, as described earlier, a high level of expert knowledge based on the experience of the employees is required. Practical experience (both previously to and during their time at ManuFact AG) is essential to master the task, which in most cases leads to a very fast cognitive processing of the requests. The following quote reflects this.

"I would say I can already answer 60 percent from my own knowledge." - Helpline Expert 2

All employees are individual knowledge carriers. However, in the case of complex or special topics (fire protection), the solution of tasks often requires an exchange of information between employees (I11). Requests that require this cognitive exchange among multiple helpline experts need to be identified by a cognitive automation solution to live up to the high service level expectations of the customers.

Relationship requirements: Overall, the relationship requirements were found to be on an intermediate level, however exhibiting a high level of volatility as in this use case in dependence on the type of inquiry and the characteristics of the customer, the requirements for relationship building (trust building etc.) vary. Furthermore, regional specifics, such as dialects and cultural differences in communicating, affect relationship-intense inquiries.

In particular this means that depending on the situation of the customer, customer behavior can vary greatly (I12). Dealing with customers is not always easy, especially when customers are under time pressure, as reflected by Helpline Expert 1.
"About two or three times a year I also have to say: 'Alright, well, let us calm down, or we have to end the conversation. "' - Helpline Expert 1

For these cases, the B2B helpline needs time, tact, and sensitivity, which requires personal human interaction.

However, often the communication can also be less complex as many inquiries are mainly about numbers, data and facts (I13). About half of all inquiries are estimated to fall into this category.

"For requests for data sheets, answers are very short and crisp. The customer won't get a love letter from us." - Helpline Head

A support of these requests through cognitive automation would be conceivable after further analysis. In that, a cognitive automation solution would need to be able to distinguish between relationship-intense (see I12) and relationship-loose customer requests.

Against this backdrop, complex enquiries demand greater communication skills from employees. In complex matters, ManuFact AG's expertise is valued and building trust plays a more significant role (I14).

"The emotional component comes into play when desperate customers call as a last resort." - Helpline Expert 1

Consequently, in complex conversations, the highest added value for the customer relationship can arise, but the greatest mistakes can also happen, which increases the requirements induced into a potential project.

Furthermore, as different customer groups have different needs and differ in communication as well as in the complexity of problems, there is not just one customer type of ManuFact AG, but several (I15). With end customers, the identification of the problem is more difficult, with experts resolving the problem is more challenging, which is described by the Helpline Head.

"The question often depends on the customer. Questions from planners and architects are more difficult and complex to solve." - Helpline Head

Thus, it will be crucial for a cognitive automation solution to detect and specify the point of handover between human and machine and vice-versa.

Finally, also cultural factors and the customer's level of knowledge affect the way the conversation is conducted (I16). Therefore, different customer groups communicate differently as indicated in this quote.

"The installer expresses himself differently." Helpline Expert 2

This also depends on regional differences (e.g. differences in dialects, terminology and culture between northern and southern regions). Besides a recognition of the customer problem, a categorization of the type of customer is essential for an individualized conversation by a cognitive automation tool, which raises the relationship requirements of the use case. 
Transparency requirements: Overall, the transparency requirements were found to be relatively low as there is no need for costly reporting or special audits other to audits that fall under the normal standard in large corporations such as ManuFact AG. Except for topics such as fire protection, the helpline experts can work on finding suitable solutions in direct interaction with the customer without third parties involved. Thus, this requirements dimension is likely to induce rather low efforts into a potential cognitive automation project. This is substantiated by the following insights.

First, for instance, the email requests are distributed evenly among the employees for processing according to the first-in-first-out principle. Apart from fire protection topics, there are no other criteria for routing or reporting the requests (I17) as described here.

"The emails are not distributed according to any specific criteria. [...] Or rather, there is one criterion: fire protection. Everything has to be legally protected and we have specially trained experts who do that." Helpline Head

However, these "critical" cases would have to be identified in advance by a cognitive automation solution to design it for process transparency.

Second, the department's reporting is only based on the number of in- and outbound communication flows. Reporting is not based on content-related criteria (I18).

"We have to count the emails manually. The phone calls are counted automatically. But we don't see what was there every day." - Helpline Expert 3

Consequently, this dimension does not lead to high additional requirements for a cognitive automation solution as the need for disclosing information to third parties outside of the helpline is kept lean.

\section{Implications of Using the Cognitive Automation Use Case Assessment Model}

Applying the use case assessment model within the customer helpline department at ManuFact AG led to several implications within the organization.

Overall, the assessment allowed the IT and business project managers to early identify stepping stones that need to be handled to achieve project success. They could seize the analysis results to enrich their line of argumentation when communicating the implications of cognitive automation endeavors at ManuFact AG to the senior management in order to prepare managerial decision making, and to manage expectations.

Against this backdrop, it was successfully communicated to the senior management that the assessed use case cannot be directly implemented in its full breadth but needs to be approached by dividing it into sub-use cases, and that work is required that needs to be conducted before an actual cognitive automation project should be approached.

Thus, the following activities were triggered at ManuFact AG as a direct implication of the assessment:

Regarding the data requirements analysis of the use case, this dimension demands a large-scale data gathering and cleaning project, which is currently planned to be rolled out on an organization-wide scale before a cognitive automation project is started.

Resulting from the variability in cognition and relationship requirements of the use case, a preceding email analysis project is planned to identify and define "standard emails". By further distinguishing sub-use cases such as automated email classification, these will be prioritized in an impact-effort matrix to provide an actionable basis for management decisions.

Finally, due to the human-centered approach of basing the assessment on interviews with the people who carry out the actual tasks and processes, the assessment was well perceived by them. This is one of the most important learnings and achievements from deploying the assessment model as cognitive automation initiatives can also lead to negative organizational feedback due to the fear of job loss that is caused by such systems. The positive reception of the assessment is reflected in this final quote.

"If we didn't have the simple emails, we would have more time for other things, that's about 30-40 percent. You would help us there if there was any solution." Helpline Expert 2

Thereby, the developed assessment model can help to early integrate internal stakeholders and to prepare them to be supportive towards cognitive automation.

\section{Concluding Remarks}

In this paper, we present a model for assessing cognitive automation use cases, which is tailored to the specifics of cognitive automation. We apply it to the organizational context in the course of an action research project at the customer service department of ManuFact AG. The insights derived from applying the model and the organizational impact at ManuFact AG showcase the applicability and utility of the developed model. Overall, the model shall help practitioners to make more informed decisions on selecting use cases for cognitive automation, and respectively plan the implementation of the latter. For research, the identified factors affecting the suitability of a use case for cognitive automation can deepen our understanding of the phenomenon of cognitive automation in particular, and $\mathrm{AI}$ as the driving force behind cognitive automation in general. 


\section{References}

[1] van der Aalst, W.M.P., M. Bichler, and A. Heinzl, "Robotic Process Automation", Business \& Information Systems Engineering 60(4), 2018, pp. 269-272.

[2] Ackoff, R.L., "From data to wisdom", Journal of applied systems analysis 16(1), 1989, pp. 3-9.

[3] Asatiani, A., and E. Penttinen, "Turning robotic process automation into commercial success - Case OpusCapita", Journal of Information Technology Teaching Cases 6(2), 2016, pp. 67-74.

[4] Autor, D.H., F. Levy, and R.J. Murnane, "The skill content of recent technological change: An empirical exploration", The Quarterly journal of economics 118(4), 2003, pp. 1279-1333.

[5] Bachrach, H.E., "Formal methods for design automation application development", 1997.

[6] Barki, H., and J. Hartwick, "Measuring user participation, user involvement, and user attitude", MIS quarterly, 1994, pp. 59-82.

[7] Baskerville, R.L., "Investigating Information Systems with Action Research", Communications of the AIS 2, 1999, pp. 1-32.

[8] Bernstein, E.S., "Making transparency transparent: The evolution of observation in management theory", Academy of Management Annals 11(1), 2017, pp. 217-266.

[9] Bologna, G., and Y. Hayashi, "Characterization of Symbolic Rules Embedded in Deep DIMLP Networks: A Challenge to Transparency of Deep Learning", Journal of Artificial Intelligence and Soft Computing Research 7(4), 2017, pp. 265-286.

[10] Bovee, M., R.P. Srivastava, and B. Mak, “A conceptual framework and belief-function approach to assessing overall information quality", International journal of intelligent systems 18(1), 2003, pp. 51-74.

[11] Bruckner, D., H. Zeilinger, and D. Dietrich, "Cognitive automation-Survey of novel artificial general intelligence methods for the automation of human technical environments", IEEE Transactions on Industrial Informatics 8(2), 2011, pp. 206-215.

[12] Butner, K., and G. Ho, "How the human-machine interchange will transform business operations", Strategy \& Leadership 47(2), 2019, pp. 25-33.

[13] Campbell, D.J., "Task complexity: A review and analysis", ACM review 13(1), 1988, pp. 40-52.

[14] Cappiello, C., C. Francalanci, and B. Pernici, "Data quality assessment from the user's perspective", International Workshop on Information Quality in Information Systems, (2004), 68-73.

[15] Davidow, M., "Organizational responses to customer complaints: What works and what doesn't', Journal of service research 5(3), 2003, pp. 225-250.

[16] Forman, J., and L. Damschroder, "Qualitative Content Analysis", In L. Jacoby and L.A. Siminoff, eds., Empirical Methods for Bioethics: A Primer (Advances in Bioethics, Volume 11). Emerald Group Publishing Ltd, 2007, 39-62. [17] Frey, C.B., and M.A. Osborne, "The future of employment: How susceptible are jobs to computerisation?", Technological forecasting and social change 114, 2017, pp. 254-280.

[18] Hosseini, M., A. Shahri, K. Phalp, and R. Ali,
"Foundations for transparency requirements engineering", International Working Conference on Requirements Engineering: Foundation for Software Quality, (2016), 225231.

[19] von Krogh, G., "Artificial Intelligence in Organizations: New Opportunities for Phenomenon-Based Theorizing", ACM Discoveries 4(4), 2018, pp. 404-409.

[20] Lacity, M., and L. Willcocks, Robotic process and cognitive automation: the next phase, SB Publishing, Ashford, UK, 2018.

[21] Lacity, M., and L. Willcocks, "Client Service Automation Deployments - What Do They Mean for Your Job and Organization?", Pulse Magazine, 2018.

[22] Leshob, A., A. Bourgouin, and L. Renard, "Towards a process analysis approach to adopt robotic process automation", 2018 IEEE 15th International Conference on eBusiness Engineering (ICEBE), (2018), 46-53.

[23] Liu, P., and Z. Li, "Task complexity: A review and conceptualization framework", International Journal of Industrial Ergonomics 42(6), 2012, pp. 553-568.

[24] Longhurst, R., "Semi-structured interviews and focus groups", Key methods in geography 3(2), 2003, pp. 143-156. [25] Louwerse, M.M., A.C. Graesser, S. Lu, and H.H. Mitchell, "Social cues in animated conversational agents", Applied Cognitive Psychology: The Official Journal of the Society for Applied Research in Memory and Cognition 19(6), 2005, pp. 693-704.

[26] Militello, L.G., and R.J.B. Hutton, “Applied cognitive task analysis (ACTA): a practitioner's toolkit for understanding cognitive task demands", Ergonomics 41(11), 1998, pp. 1618-1641.

[27] Pipino, L.L., Y.W. Lee, and R.Y. Wang, "Data quality assessment", Communications of the ACM 45(4), 2002, pp. 211-218.

[28] Poosapati, V., V.K. Manda, and V. Katneni, "Cognitive Automation Opportunities, Challenges and Applications", Journal of Computer Engineering and Technology 9(5), 2018, pp. 89-95.

[29] Rahwan, I., M. Cebrian, N. Obradovich, et al., "Machine behaviour", Nature 568(7753), 2019, pp. 477-486.

[30] Rudowsky, I., "Intelligent agents", The Communications of the AIS 14(1), 2004, pp. 275-290.

[31] Short, J., E. Williams, and B. Christie, The social psychology of telecommunications, Wiley \& Sons, 1976.

[32] Strong, D.M., Y.W. Lee, and R.Y. Wang, "Data quality in context", Communications of the ACM 40(5), 1997, pp. $103-110$.

[33] Syed, R., S. Suriadi, M. Adams, et al., "Robotic Process Automation: Contemporary themes and challenges",

Computers in Industry 115, 2020, pp. 1-15.

[34] Theodorou, A., R.H. Wortham, and J.J. Bryson, "Why is my robot behaving like that? Designing transparency for real time inspection of autonomous robots", AISB 2016, 2016, pp. 63-66.

[35] Zarkadakis, G., R. Jesuthasan, and T. Malcolm, "The 3 ways work can be automated", Harvard Business Review, 2016. 